\title{
Challenges of Implementation of e-learning in Mathematics, Science and Technology Education (MSTE) in African schools: A Critical Review
}

\author{
Samson O. Gunga \\ University of Nairobi \\ gungasamson@gmail.com
}

\begin{abstract}
This paper discusses the general ICT challenges in education and poses questions, the attempt of whose answers establish the manner in which e-learning technology could be appropriate for understanding and communicating the structures of mathematics and science. Challenges in understanding mathematics and science arise out of the interaction between these two intertwined yet disparate disciplines. While mathematical proof is established deductively and hence conclusive and not amenable to confutation in a logically possible world, scientific truth is established inductively on probable yet utilitarian grounds in the actual world. While challenges in implementation of the understanding of mathematics and science through technology arise from social and infrastructural issues related to ICT in African environment, the difficulty posed by challenges of communicating the principles of understanding the structure of mathematics and science are not yet insurmountable. An attempt to bring into coherence the mathematical and scientific understanding through e-learning instructional paradigm in quasi-philosophic terms is the main subject of this paper.
\end{abstract}

\section{Introduction}

E-learning, broadly defined is the use of electronic technology to deliver education and training applications, monitor learner's performance and report learner's progress. It is defined as an innovative approach for delivering electronically mediated, well-designed, learner-centered and interactive learning environments to anyone, anyplace, anytime by utilizing the internet and digital technologies (Hedge \& Hayward, 2004). However, it should be noted that instructional methods employed during teacher-learner interchange are constrained by the internal structure of a discipline. Therefore a study of the technical language and structure of disciplines is always at the heart of the problem of transfer even in e-learning. Beliefs about how mathematical and scientific language may be used to understand their structural principles have direct influence on the implementation of effective e-learning of the same. This is an area that poses immense challenge as it points to issues that call for methodological revolution that has not been envisaged. The study of the language and structure of disciplines and how to communicate such structure has not taken root in mathematics and scientific research.

\section{Challenges in the revolutionary development of Information and Communication Technologies}

While the urge to embark on e-learning is high in Africa, it is still a dream because of weak ICT infrastructure, unsensitized populace and technophobic work force. The wide gap between the experiences of ICT experts who develop e-learning systems and the slow, and sometimes unwilling, designers of education and instructional systems planners is a challenge

Journal of Contemporary Issues in Education, 2010, 5(1), pp.45-51

ISSN 1718-4770 () 2010 University of Alberta

http://ojs.educ.ualberta.ca/index.php/jcie/ 
that ought to be bridged through sensitization and training. Some of the challenges of e-learning in African schools are the following:

1. Non inclusion of regular 'technology education' and 'technology teacher education' as core requirement in many systems of education in African countries.

2. Getting stuck in traditional teaching methodology due to static national policy on ICT, technology (teacher) education and digital inclusion.

3. Inadequate research and hence ignorance on the understanding and communication of the structure of mathematics.

4. Although Mobile Technology is embraced widely, it is associated with high cost of usage, small screen size and low life of battery.

5. The cost of personal computer, laptop, software, internet and their technical support is high.

6. Unsensitized students and staff are stuck to face to face pedagogy in many times.

7. The cost of access to Internet is still very high in most African countries.

8. Problem of power instability, blackouts in towns and unconnectedness in rural areas is challenging.

The above challenges exist for almost all categories of people who intend to use ICT in any area of education. The implementation of e-learning in mathematics and science face a double challenge emanating from the nature of the disciplines. The question that this paper grapples with, and that which researchers ought to focus on relate to the following:

(a) Elucidation and understanding of the structure of mathematics and sciences

(i) How is the structure of mathematics discerned?

(ii) What does mathematization of the sciences entail?

(b) What is the optimum way of communicating the structure of mathematics and science in education?

(c) What is the understanding behind the application of the logic of mathematics and science in socio-cultural settings and socialization process?

(d) What principles can be used for the sustaining of personal confidence, questioning and optimistic attitude in the teaching and learning of mathematics and science?

(e) How does ICT capture the mood of understanding and communicating the structure of mathematics?

\section{Concept of structure}

The concept of structure evokes certain associations. The significance of mathematics and science may not be perceived unless and until each is integrated into what it forms a part of. Paradoxically, the whole (mathematics or science) may also not be effectively perceived unless the elements that constitute it are seen to meaningfully cohere within a unitary context. It is this interaction between the two paradigms within a discipline that yields its structure. A structure consists of elements and law-like relations between such elements which form a thought that has a logic, texture and relevant background insights peculiar to the discipline and which necessitates and determines the internal ordering that constitutes its unique structure. The structure expresses wholeness (intrinsic orderliness), transformation (structured and structuring laws of intelligible 
change that preserves invariance in certain respects), and self-regulation (internal coherence that conform to a set of intrinsic laws which ensures no appeal beyond itself in order to validate its transformational procedures).

\section{Perception of the structure of mathematics and science}

The study of the structure of disciplines is an area that has not been satisfactorily explored. Theories about the origin and structure of mathematics and science could be of real interest to philosophers and educators alike. Through Bruner (1960), the idea of structure of a discipline became known. Conception of scientific data seems to be quasi logico-mathematical and includes what can be measured and revealed through observation, experimentation and intuitive logical aid to man's epistmetic powers. Although the aids are normally in the form of charts, diagrams, video tapes, concrete and mental models (Abimbola \& Danmole, 1995), it is mathematics that attempts to bridge the gap between scientific concreteness and abstraction. In science, just like in mathematics, the study of ideas moves from classificatory, comparative to quantitative and this is what creates difficulty in mathematical-scientific understanding.

Scientific conclusions are largely inductive and probable while mathematical proof upon which scientific knowledge is grounded is deductive, conclusive and non-confutable. Probably this is the basis for Kuhn's paradigm shift. Kuhn's analysis of how scientists explore the world and make discoveries rejects the notion that research methods are rational and objective, instead arguing that throughout the history of science, paradigm shifts in knowledge and evidence cause major changes and radical alterations (Zoltan, 2010) contrary to mathematical truths that are conclusive and perennial.

\section{The interaction between mathematics and science}

In attempt to actualize his understanding, man establishes a natural link between mathematics and the physical and social sciences. He does this by developing a synthesis of content strands derived from interacting levels of the structure of mathematics. It is therefore, apparent that the root of mathematics inheres in perceptions, being the ordering of experiences, objects and events from the phenomenal world of patterns. An attempt to describe a mathematical or scientific system is constrained by appeal to principles of philosophy of mathematics or science. For instance, although mathematics, as a self-contained discipline has nothing to do with science, the latter cannot do without mathematics. Mathematics has an inherent practical utility since it is capable of capturing the laws of the material world and thus serves as a powerful instrument for our knowledge and mastery of nature. Mathematics reveals order in the physical universe, and its importance in curricula arise from its inherent power to describe, explain and predict trends in phenomena. The practical utility of mathematics reveals it as a system for problem solving and improvement of human condition which science endeavours to achieve.

The first mathematical experiences are basically intuitive, generic and utilitarian. Intuition from simple representations such as dealing with numbers taken from real life situations, with fingers, coins, measures, weights etc., makes children start mathematical development with decisive influence. They quickly recognize the utilitarian value of mathematics which comes from the fact that it imposes or detects order in our interpretation of the world. An experience of such essential extrinsic value of mathematics is actualized by raising 
the measurable performance of children with regard to numeracy, recognition of patterns and necessary or contingent relationships.

Any experience starts with people's attempt to identify the experience by 'name'. Invariably, it is after naming that people tend to focus on the properties of any particular experience. We identify seven modes of categorization of the properties of people's experiences which structure perception both mathematically and scientifically as discussed by Liebeck (1984) and Graham (1985) in their separate works:

\begin{tabular}{|l|l|}
\hline Matching & Selection of properties common to a range of experiences \\
\hline $\begin{array}{l}\text { Sorting } \\
\text { (Classifying) }\end{array}$ & Breaking up a matching set into new sets of uniquely matching individuals \\
\hline Pairing & $\begin{array}{l}\text { Development of a one-to-one correspondence between experiences of certain } \\
\text { specified characteristics. }\end{array}$ \\
\hline Counting & $\begin{array}{l}\text { Recognition of quantitative properties exhibited by experiences - properties } \\
\text { such as unity, pairs and multiplicity }\end{array}$ \\
\hline Ordering & Arrangement of experiences according to a specified rule of size \\
\hline Comparing & $\begin{array}{l}\text { Recognition of similarity in numbers, shape, size or position between two } \\
\text { experiences }\end{array}$ \\
\hline Measuring & Identification of the quantitative states of a property within a given experience \\
\hline
\end{tabular}

\section{Abstraction and the logic of the interaction of possibility and actuality}

The development of mathematics is based on a continual attempt to harmonize the apparent conflict between actual and logically possible existence, or between the concrete and the abstract which seem to define the relationship between mathematics and science. In fact, paradoxically, it is the grappling with the apparent conflict between abstraction from actuality and abstraction from possibility that has led to the development of mathematical and scientific systems.

The conflict between actuality and possibility abound: discrete versus continuous; particular versus general; change versus permanence; material versus formal; finite versus infinite; real versus ideal and so on. Attempts to resolve these conflicts develop the mathematical and scientific systems and reveal mathematics in particular as a conceptual system in the following sense that Bunge (1985) describes:

1. Mathematics does not make necessary use of empirical data or procedure

2. Mathematics posits or conjectures the laws (general patterns) satisfied by members of the conceptual system

3. Mathematics proves or disproves conclusively the conjectures it posits

To explain the above inherent nature of mathematics described by Bunge, it is important to assume that while concepts in the general sense arise out of perceptions (actual acquaintance with experiences and engaging in actions of various kinds), in mathematics, conceptions are idealized into a hierarchy of abstractions generated from axioms and postulates which are the logical and systematic idealizations of perceived facts.

In mathematics, conception is the ordering of perceptual knowledge into knowledge of relationships that cut across individual particulars to embrace the universal principle of 
connectedness, which is governed by the laws of logic expressed through argumentation and computation. Only slowly do children realize, for instance, that four persons, four stones, four oranges and so on do have something in common - their 'fourness' - from which we abstract the number concept of four. Once children realize that ' 4 ' is something more than a way of counting things, they develop an awareness that numbers have a use of their own, and do not always have to be connected with actual things, but 'possible things' of which actual things are, logically, a subset.

Mathematical language as the medium for describing and analyzing possible world existents has been aptly discussed by Cooley:

Mathematicians can often draw conclusions about situations in which direct investigation is impossible. Using abstractions based on physical observations, the mathematician calculates the mass of the sun while sitting at his desk.

Because mathematics can go wherever reason may lead, it has enabled scientists to find explanations of phenomena which could not be discovered by observation. Gravitation, for example, can be explained as a property of a space we cannot perceive. This space is a mathematically constructed model which satisfies all conditions an explanation of gravity requires (Cooley, 1968).

By having the power to investigate beyond the perceptual givens into the realm of logical possibilities where direct perception fails, mathematics broadens and clarifies the scope of scientific operations. It is probably in this regard that mathematics is often referred to as 'the language of the sciences'. Mathematics therefore establishes a dialectical relationship between 'abstractions from actuality' - the scientific way and 'abstraction from possibility'; it is this dialogue - 'mathematical dialectics' - which forms the basis for mathematical discourse.

\section{Understanding of mathematico-scientific issues and problem solution}

An adequate understanding of problem representation is a prerequisite for successful problem solution, the latter being a succession or sequence of problem states which terminate with a goal state, such that each successive state is obtained from the preceding states by means of an allowable logical action. A solution procedure is an ordered succession of events which involve building sub-goals with a range of possible given materials and operations. The materials and operations have to satisfy some constraints specified in the problem situation. Problem solving procedure may be ordered as follows:

(a) Identification of givens and the goal

(b) Identification and attainment of sub-goals

(c) Performance of operations and transformations toward solution

It is helpful to have a detailed representation and understanding of the goal. This may be done by increasing the specificity of the goal by deriving its additional properties using either the statement of the properties of the goal as given in the original problem, or by using given information to derive properties of the goal. The purpose of increasing the specification of the goal is to introduce the necessary working concepts for reaching it, which in turn reveals the necessary sub-goals. 


\section{Communication of mathematical and scientific experience in education}

The basic concern in this study has been a search of a philosophy of mathematics and science education which utilizes structuralism as the theory of focus for understanding and communication of the structure of the two disciples through technology. One of the objectives of the study which we have achieved variously is to give reasons why curricula formulation for communicating the structure of a discipline through technology education, should not only concentrate on individual mathematical or scientific entities but also explicate the structural features of mathematical and scientific systems through an analysis of their language. The central problem of any philosophy of mathematics and science education is the issue of the relationship between philosophy of mathematics, philosophy of science and pedagogy. In this case, it is the concern with translation of the structure of mathematics and science through the language of a discipline, into instructional procedures that create an understanding of a problem situation in an instructional setup and consequently, the formulation of problem solving strategy.

The question we have attempted to grapple with is: to what extent is 'Mathematical and scientific Language' appropriate for pedagogic communication of the structure of mathematics and science. It is noteworthy that communication of mathematical and scientific principles has been a continued exercise in man's life through the ages and has to embrace technology in the age of globalization. Man has had interest in recording and duplicating natural patterns, regularities and sequences evident in nature, artistic designs, art forms etc. All these practices have sub-consciously utilized interacting principles that regulate the working of the levels of the structure of mathematics and science. For instance, the roots of mathematics lie deep in the problems and issues arising from the phenomenal world that the scientist grapples with, but elements of abstraction and of generalization do enable mathematical studies to evolve independently of such problems.

\section{Conclusion}

If we may ask: what is it that activates man's epistemic powers with regard to the discernment of mathematical and scientific intelligibility through technology? The answer to this question lies in mathematical, scientific and philosophical research. The aids to the power of human reasoning range from appeal to extra-logical modes of inquiry such as employment of concrete, semiconcrete and analogical models through tautological transformations such as calculations to rigorous proofs. Although computation tends to dominate low level mathematics, for instance, the mathematical system does not seem to have grown solely out of the need to compute using intuitive entities that scientists manipulate and describe. Instead, it seems to have grown out of people's rational capacity to categorize experience in terms of sets of similarity and difference relations that characterize both the properties of visible and intelligible worlds. The challenge then is, if understanding of science and mathematics are a challenge in ordinary pedagogy, it would require a renewed revolutionary approach to implement effective e-learning mathematics and science pedagogy in African nations. 


\section{References}

Abimbola O. \& Bolanle T. D. (1995). Origin and structure of science knowledge: Implications for concept difficulty in science. Ilorin Journal of Education, 15, pp.46-58. Retrieved March 17 from http://www.unilorin.edu.ng/unilorin/publications/abimbola/ORIGIN\%20AND\%20STRUCT URE\%20OF\%20SCIENCE\%20KNOWLEDGE.htm

Bruner, J. S. (1960). The process of education. Cambridge, Massachusetts: Harvard University Press.

Bunge M. (1985). Treatise on basic philosophy Vol. 7: Epistemology \& Methodology. Dordrecht: D. Reidel Publishing Co., p. 22.

Cooley H. R. (1968) \& Howard E. Wahlert, Introduction to mathematics, 2nd ed. Boston: Honghton Mifflin Company, p. 444.

Graham A. T. (1985). Help your child with maths. London: Fontana Paperbacks.

Hedge, N. and Hayward, L. (2004). Redefining roles. University e-learning contributing to Lifelong learning in a networked world. E-Learning, VOL. 1, $128-145$

Liebeck P. (1984)). How children learn mathematics: A guide for parents and teachers. Middlesex: Penguine Books.

Zoltan, M. (2010). The Structure of Scientific Revolutions: Thomas S. Kuhn's Theory of Paradigm $\quad$ Shifts. $\quad$ Retrieved $\quad$ March 17 from http://philosophybooks.suite101.com/article.cfm/the_structure_of_scientific_revolutions 